\title{
Knee Examination under Anesthesia: Development of a Predictive Score for Partial Anterior Cruciate Ligament Tears
}

\author{
Max Ekdahl, $\mathrm{MD}^{1,2}$, Marcelo Acevedo, $\mathrm{MD}^{1}$, Cristian Dominguez, $\mathrm{MD}^{1}$, Maximiliano Barahona, $\mathrm{MD}^{1,3}$, \\ Rodrigo Hernandez, $\mathrm{MD}^{1}$, and Ignacio Mujica, $\mathrm{MD}^{1}$ \\ ${ }^{1}$ Department of Orthopedic Surgery, Clinica Indisa, Santigo; ${ }^{2}$ Department of Orthopedic Surgery, Clinica Las Condes, Santigo; ${ }^{3}$ Department of Orthopedic Surgery, \\ Hospital Clinico Universidad de Chile, Santigo, Chile
}

\begin{abstract}
Purpose: To determine the accuracy of knee examination under anesthesia (EUA) and develop a prognostic score for partial anterior cruciate ligament (ACL) tears.

Materials and Methods: A total of 229 patients with an ACL injury were included. Knee EUA was performed using the Lachman test, pivot shift test and arthrometric maximum manual side-to-side difference (AMMD) test. The arthroscopic examination is the gold standard for the diagnosis of partial and complete ACL tears, which was compared with EUA findings. Multivariate logistic regression was estimated, and the significant variables were used to develop a predictive score.

Results: The relative risk for a complete tear with Lachman 2+ was 8.55 (range, 3.5 to 20.7) and 53.04 (range, 6.7 to 417) with Lachman 3+, compared to Lachman 1+. Negative pivot shift was reported in 23 cases in the partial tear group (76.7\%) and in 22 in the complete tear group (11.1\%). The AMMD was $3.5 \mathrm{~mm}$ in the partial tear group and $5.4 \mathrm{~mm}$ in the complete tear group $(\mathrm{p}<0.05)$. A prognostic score of less than five suggested the presence of a partial ACL tear. The score showed $81.1 \%$ sensitivity and $68.7 \%$ specificity.

Conclusions: Partial ACL tears can be differentiated from complete tears with Lachman test, pivot shift test, and AMMD test.
\end{abstract}

Keywords: Knee, Anterior cruciate ligament, Injury, Partial, Examination

\section{Introduction}

The anterior cruciate ligament (ACL) consists of two anatomic and functional bundles, the anteromedial (AM), responsible for stabilizing the knee joint in response to anterior tibial load, and the posterolateral (PL), responsible for stabilizing the knee in combined rotatory load ${ }^{1)}$. Recent interest in anatomic double-

Received January 23, 2018; Revised (1st) April 6, 2018;

(2nd) April 24, 2018; Accepted April 25, 2018

Correspondence to: Maximiliano Barahona, MD

Department of Orthopedic Surgery, Clinica Indisa and Hospital

Clinico Universidad de Chile, Santiago Chile1810 Santa María Avenue,

Providencia, Santiago 7520440, Chile

Tel: +56-9-99791493, Fax: +56-2029788225

E-mail: maxbarahonavasquez@gmail.com

This is an Open Access article distributed under the terms of the Creative Commons Attribution Non-Commercial License (http://creativecommons.org/licenses/by-nc/4.0/) which permits unrestricted non-commercial use, distribution, and reproduction in any medium, provided the original work is properly cited. bundle ACL reconstruction has increased attention to partial ACL ruptures and augmentation techniques, preserving tissue remnants. Several reports described the clinical outcomes of ACL reconstruction preserving the bundle that remains intact ${ }^{2-6)}$. Theoretical advantages of preserving ACL tissue include proprioception $^{7)}$, improved graft vascularity and biomechanical properties $^{5,8)}$. The decision to preserve ACL tissue is challenging and determining if the remaining tissue is biomechanically functional is difficult. Clinical and arthrometric examination under anesthesia (EUA) in combination with arthroscopic assessment can be used as a tool for this purpose. There is, however, a lack of information regarding findings in the physical examination in partial ACL ruptures. Magnetic resonance imaging (MRI) is an excellent diagnostic test for ACL tears; however, in the case of a partial tear, it is not useful in determining whether to preserve remnant fibers or not since it does not show the functional state.

The purpose of this study is to determine the accuracy of knee EUA and develop a prognostic score for the diagnosis of a par- 
tial ACL tear. We hypothesized that complete tears would have higher Lachman test grades, greater pivot shift test grades and higher arthrometric maximum manual side-to-side differences (AMMD) compared to partial ACL ruptures.

\section{Materials and Methods}

A cohort study was designed and approved by our Institution's Ethics Review Board. All participants provided informed consent. The participants were all consecutive patients who consulted with the physician (ME, MA, and RH) between May 2007 and May 2012 for acute traumatic knee or persistent sensation of instability and a positive Lachman test. A torn ACL was suspected and confirmed with an MRI in them, and they underwent primary ACL reconstruction. Patients were excluded if they had two or multi-ligament knee injuries, previous ACL reconstruction, contralateral ligament knee injuries or contralateral ACL reconstruction.

A total of 229 patients were recruited. No attempt to differentiate partial from complete ACL ruptures in MRI was made. All of them were examined under anesthesia before surgery. The knees were assessed with the patients under spinal anesthesia and before sterile skin preparation. The same examiner, a senior orthopedic surgeon, performed all evaluations. The Lachman test was graded as $1+$ if anterior tibial translation was subjectively estimat- ed to be less than $5 \mathrm{~mm}$; as $2+$, between $5-10 \mathrm{~mm}$; or 3+, greater than $10 \mathrm{~mm}$. The pivot shift test was graded as $1+$ if a subtle change of motion or glide without an appreciated articular clunk was present; a 2+ pivot shift represented a distinct clunk; and a $3+$ pivot shift represented a gross clunk or locking in the subluxated position ${ }^{9)}$. Anterior displacement was measured by the KT1000 knee arthrometer (MEDmetric, San Diego, CA, USA) with the knee at $20^{\circ}$ of flexion, applying a maximum manual anterior force. Both the involved and uninvolved knees were assessed, and the AMMD in anterior tibial displacement was registered. The results of physical examination were compared with arthroscopic findings. The arthroscopic evaluation was performed via the AM, anterolateral and accessory AM portals. Any accompanying meniscal or chondral lesions were treated. A partial ACL tear was defined as a tear of one of the two ACL bundles (AM or PL). The partial ACL rupture was confirmed and the quality of the remaining tissue was subsequently evaluated (Fig. 1). The remnant bundle was meticulously probed, and its tension pattern was assessed through the whole knee range of motion. When the remnant bundle was considered anatomically intact, an augmentation technique was performed with a hamstring graft (Fig. 2). If a patient had a complete ACL tear, single- bundle anatomic reconstruction was performed using a quadruple hamstring graft, and the femoral tunnel was drilled through an AM accessory portal. Cortical fixation of the femur was performed and a screw

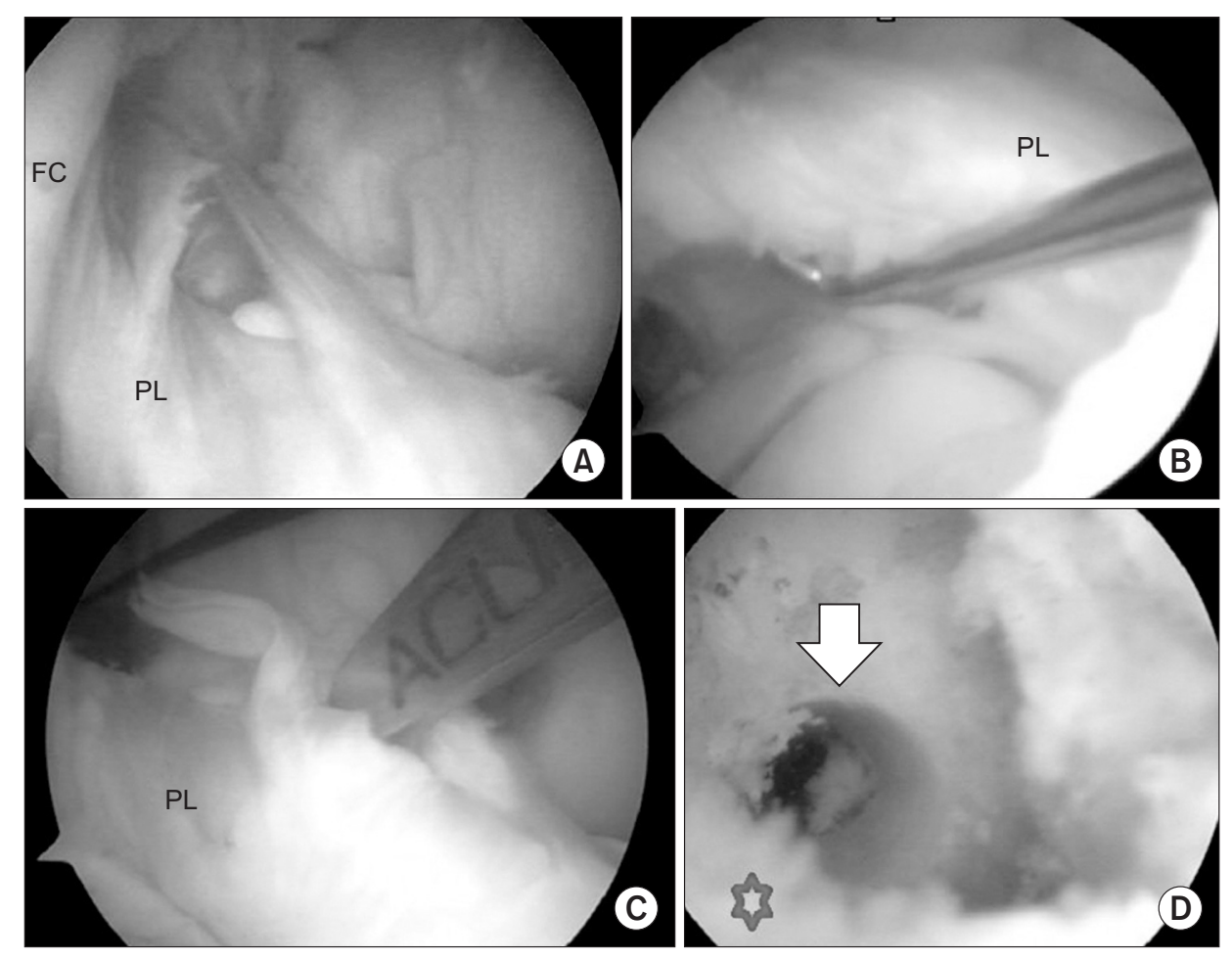

Fig. 1. Partial anterior cruciate ligament (ACL) tear reconstruction. (A) Arthroscopic view from the anterolateral portal. The posterolateral (PL) bundle seemed healthy and attached to the lateral femoral condyle (FC). (B) In the arthroscopic examination, it seemed that the PL had good tension. (C) The PL bundle was preserved. A tunnel was made at the femoral anteromedial bundle footprint. A tibial guide was positioned for tibial tunnel drilling. (D) The white arrow indicates femoral tunnel, and the star indicates the PL bundle. 
was used for tibial fixation.

For statistical analysis, Wilcoxon nonparametric unpaired test was used to assess the difference in KT-1000 measurement between patients with a complete tear (complete tear group) and a partial tear (partial tear group). The relative risk (RR) for the type of ACL injury was calculated using a nominal regression model for the Lachman test and pivot shift test using the lowest grade of each test as the reference. Then, multivariate logistic regression was performed, and the significant variables were used to develop a predictive score. Binomial regression was used to weigh each variable in the score. Univariate logistic regression was estimated,

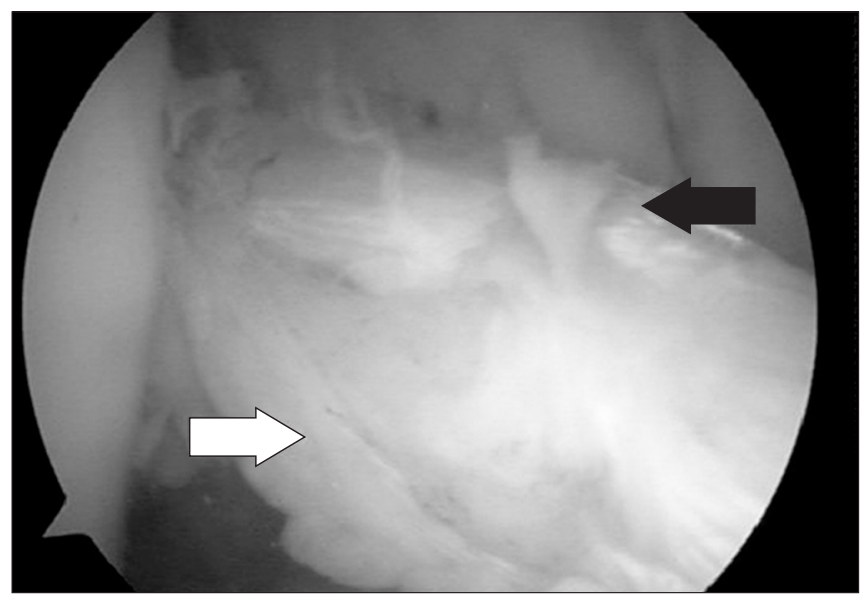

Fig. 2. Final result of anterior cruciate ligament reconstruction (black arrow) with preservation of the posterolateral bundle (white arrow).

Table 1. Physical Examination Results according to Anterior Cruciate Ligament (ACL) Tear Type

\begin{tabular}{cccc}
$\begin{array}{c}\text { Exam/ACL } \\
\text { injury }\end{array}$ & $\begin{array}{c}\text { Partial } \\
(\mathrm{n}=30)\end{array}$ & $\begin{array}{c}\text { Complete } \\
(\mathrm{n}=199)\end{array}$ & $\begin{array}{c}\text { Relative risk } \\
(95 \% \mathrm{CI})^{\mathrm{a})}\end{array}$ \\
\hline $\begin{array}{c}\text { Lachman } \\
1+\end{array}$ & $19(63.33)$ & $24(12.06)$ & \\
$2+$ & $10(33.34)$ & $108(54.27)$ & $8.55(3.53-20.70)$ \\
$3+$ & $1(3.33)$ & $67(33.67)$ & $53.04(6.73-417.92)$ \\
Pivot shift ${ }^{\mathrm{b})}$ & & & \\
0 & $23(76.67)$ & $22(11.06)$ & \\
$1+$ & $7(23.33)$ & $80(40.20)$ & $11.95(4.53-31.48)$ \\
$2+$ & 0 & $71(35.68)$ & - \\
$3+$ & 0 & $26(13.07)$ & - \\
\hline
\end{tabular}

Values are presented as number (\%).

CI: confidence interval.

${ }^{\text {a) }}$ Relative risks were calculated using Lachman $1+$ and pivot shift 0 as reference points.

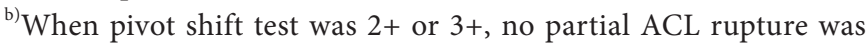
observed. using the score as the independent variable and ACL injury as the dependent. Then, a receiver operating characteristic (ROC) curve was estimated, and the cutoff point was determined using maximum likelihood optimization. Confidence intervals of $95 \%$ and $5 \%$ significance levels were calculated. Data were processed using Stata ver. 11.2 (StataCorp., College Station, TX, USA).

\section{Results}

The average cohort age was 33.2 years (range, 15 to 60 years) with a female to male ratio of 0.8 . After arthroscopic evaluation, partial ACL ruptures were confirmed in 30 cases $(13.1 \%)$, of which $25(83.3 \%)$ corresponded to AM bundle ruptures and 5 (16.7\%) corresponded to PL bundle ruptures.

Results of Lachman and pivot shift tests matched with arthroscopic findings are shown in Table 1. Of note is that higher grades in both Lachman and pivot shift tests were associated with the greater likelihood of finding a complete rupture in the arthroscopy. A negative pivot shift test was reported in 23 cases in the partial tear group (76.7\%) and in 22 cases in the complete tear group (11.1\%). When a pivot shift test $2+$ or $3+$ was found, no partial ACL rupture was observed. The mean AMMD was 3.53 $\mathrm{mm}$ in the partial tear group and $5.46 \mathrm{~mm}$ in the complete tear group. The median AMMD in the partial tear group was $3.5 \mathrm{~mm}$ (range, 2.32 to $4.68 \mathrm{~mm}$ ), and in the complete tear group, it was 5 $\mathrm{mm}$ (range, 4.59 to $5.41 \mathrm{~mm}$ ) ( $\mathrm{p}=0.01$ ) (Fig. 3). A firm endpoint was present in $100 \%$ of the partial tear group and in $21 \%$ of the complete tear group.

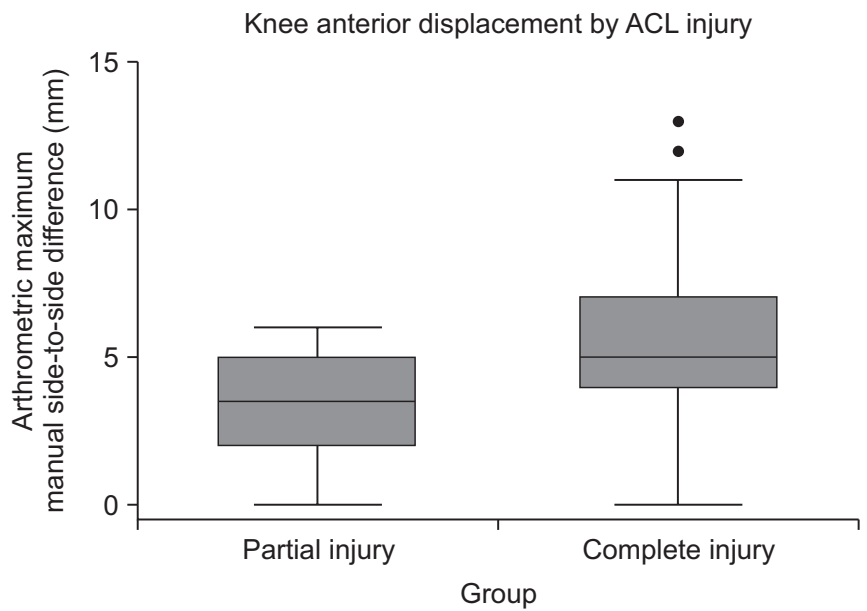

Fig. 3. The arthrometric maximum manual side-to-side difference between partial and complete anterior cruciate ligament (ACL) tear groups. The median value in the partial tear group was $3.5 \mathrm{~mm}$ (range, 2.32 to $4.68 \mathrm{~mm}$ ) and the value in the complete tear group was $5 \mathrm{~mm}$ (range, 4.59 to $5.41 \mathrm{~mm}$ ). 
Table 2. Results of Multivariate Logistic Regression Analysis

\begin{tabular}{lcc}
\hline \multicolumn{1}{c}{ Variable } & Odds ratio (95\% CI) & p-value \\
\hline Pivot shift & $3.4(1.1-10.7)$ & 0.03 \\
Lachman & $10.2(1.9-54.2)$ & 0.01 \\
Arthrometric measurement & $1.61(1.1-2.3)$ & 0.01 \\
\hline
\end{tabular}

CI: confidence interval.

Table 3. Scoring Method for Physical Examination Finding to Calculate the Predictive Score of Partial Anterior Cruciate Ligament Tears

\begin{tabular}{lc}
\hline \multicolumn{1}{c}{ Test } & Point \\
\hline Lachman test 1+ & 0 \\
Lachman test 2+ or 3+ & 1 \\
Pivot shift test $1+$ & 1 \\
Pivot shift test 2+ & 2 \\
Pivot shift test 3+ & 3 \\
Arthrometric measurement & mm=points \\
\hline
\end{tabular}

Multivariate logistic regression results showed that the pivot shift test $(p=0.03)$, Lachman test $(p=0.01)$ and AMMD $(p=0.01)$ test are significant predictive variables for a partial ACL tear. The Lachman test was more significant when the variable was divided into Lachman 1+ and the rest, 2+ and 3+ (Table 2).

The predictive score was developed with those three variables: Lachman test $1+$ scores 0 point and Lachman test $2+$ or $3+$ scores 1 point; pivot shift test $1+$ scores 1 point, pivot shift $2+$ scores 2 points and pivot shift $3+$ scores 3 points. AMMD was scored according to their net value: if an AMMD was $3 \mathrm{~mm}$, the score was 3 points and if it was $2 \mathrm{~mm}$, the score was 2 points. Accordingly, for example, a patient with Lachman $2+$, pivot shift $2+$ and AMMD of $8 \mathrm{~mm}$ scores 11 points $(1+2+8)$ (Table 3$)$. The predictive score had an odds ratio of 1.95 (range, 1.43 to 2.66) and an ROC curve with the area under the curve of 0.86 (range, 0.77 to 0.94) (Fig. 4). If a patient has a total score of less than five points, it suggests a partial ACL tear, with a sensitivity of $81.8 \%$, specificity of $68.7 \%$, a positive predictive value of $95.6 \%$ and a negative predictive value of $31.3 \%$.

\section{Discussion}

The recent interest in anatomic ACL reconstruction has been focused on partial ACL ruptures and augmentation techniques preserving ACL tissue or an intact ACL bundle. However, the decision to preserve tissue is complicated, and there is a lack of information regarding clinical and arthrometric evaluation in partial ACL ruptures. The purpose of the present study was to

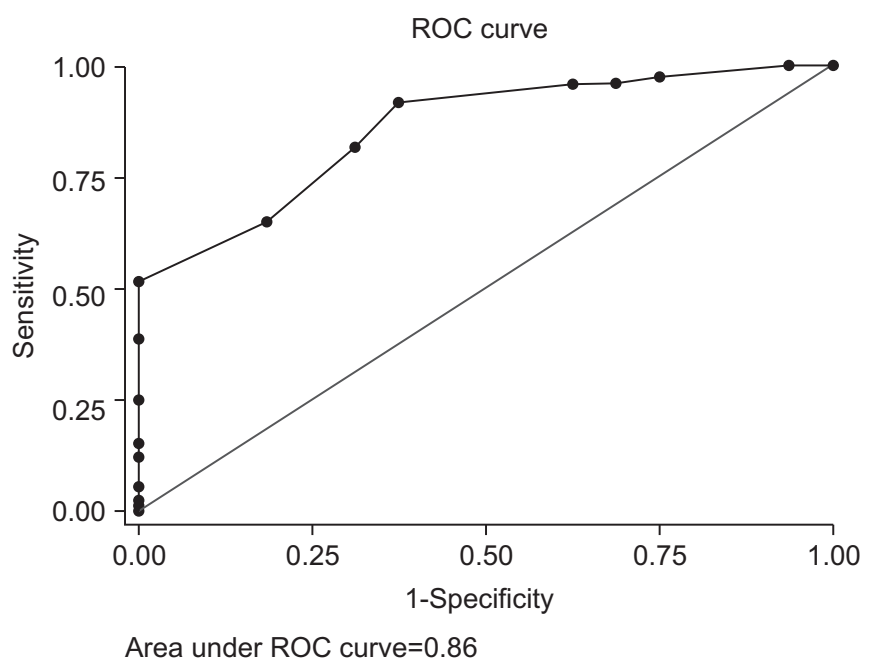

Fig. 4. Receiver operating characteristic (ROC) curve for the predictive score. The area under the curve was 0.86 (range, 0.77 to 0.94 ).

determine if the clinical and arthrometric preoperative knee EUA is helpful in the diagnosis of a partial ACL rupture. Our hypothesis was confirmed. We found statically significant differences in the Lachman test, pivot shift test and the AMMD between partial and complete ACL ruptures.

Arthrometric evaluation using a KT-1000 arthrometer is the most commonly used ligament test, and particularly the maximum manual test has shown to be the most reliable testing method to discriminate normal from pathologic anterior knee laxity ${ }^{10)}$. According to our findings, a low grade side-to-side difference in arthrometric evaluations suggests a partial ACL rupture. Similar findings are reported in the literature ${ }^{6,11-14)}$. In a case series study of isolated AM and PL bundle augmentation, Ochi et al. ${ }^{11)}$ reported a preoperative side-to-side difference of anterior displacement measured by the KT-2000 knee arthrometer at $30 \mathrm{lb}$ as $3.4 \pm 2.4 \mathrm{~mm}$; however, a comparison with complete ACL ruptures was not performed. In a case series of partial ACL ruptures, Fritschy et al. ${ }^{12)}$ reported a consistent maximum manual side-to-side difference that never exceeded $3 \mathrm{~mm}$. Similar results were also shown by Bak et al. ${ }^{13)}$ in a case series of partial ACL ruptures reporting a side-to-side difference of less than $3 \mathrm{~mm}$ in 24 out of 34 cases. Sonnery-Cottet et al. ${ }^{14)}$ reported a side-to-side difference of $4.8 \mathrm{~mm}$ in partial ACL ruptures (PL bundle tears) compared to $7.5 \mathrm{~mm}$ in complete tears. A delayed firm endpoint was reported in all cases with a partial rupture. Adachi et al. ${ }^{6}$ in a comparative study showed that the preoperative side-to-side difference of anterior displacement measured by the KT-2000 knee arthrometer at $30 \mathrm{lb}$ was $5.3 \mathrm{~mm}$ in a partial ACL rupture group and $6.0 \mathrm{~mm}$ in a complete ACL tear group. 
A non-graduated preoperative pivot shift was reported as positive in $93.3 \%$ in the partial tear group and $92 \%$ in the complete tear group. The differences were not significant. Recently, Dejour et al. ${ }^{15)}$ reported similar findings in a case-control study comparing the same clinical tests, MRI and instrumented laxity using the Telos Stress Device (Telos GmbH, Marburg, Germany). In their results, pivot shift test $2+$ or $3+$ was consistent with complete ACL ruptures and pivot shift test negative or $1+$ was related to partial ruptures. The mean side-to-side difference was $9.1 \mathrm{~mm}$ for complete ruptures and $5.2 \mathrm{~mm}$ for partial ruptures. Although differences between groups were also reported significant, their values are higher than our results. The different devices used to measure anterior tibial translation may explain the difference. In a retrospective study, Yoon et al. ${ }^{16)}$ compared the preoperative findings in physical examination and MRI among standard ACL reconstruction, AM bundle augmentation and PL bundle augmentation. No differences in the anterior drawer test and Lachman test among groups were reported, but the AM bundle augmentation group showed a lower incidence of positive pivot shift test than the standard ACL reconstruction group and PL bundle augmentation group, suggesting that the presence of an entire PL bundle is related to a negative pivot shift finding. Because the number of patients with an isolated PL bundle rupture was too small (5 patients) in the current study, we did not attempt to differentiate knee EUA between partial AM and PL bundle ruptures, and we grouped them for analysis purposes into a partial tear group. The RR of having a complete tear with pivot shift $1+$ was 11.9, and no partial ruptures were presented with pivot shift $2+$ or $3+$. Our findings suggest a high correlation between positive pivot shift test and a complete ACL rupture. In other words, the pivot shift test is relevant when its value is positive and not when it is negative for the diagnosis of a partial ACL rupture.

There are several limitations to consider when interpreting the results of this study. The evaluation of Lachman's endpoint and pivot shift testing is subjective; we did not assess intraobserver or interobserver agreement, and the anterior tibial translation might not be the most accurate method to detect differences in partial versus complete ACL ruptures. In a computer model, Liu et al. ${ }^{17)}$ showed that stiffness and the rate of change of stiffness of the anterior force displacement better predict partial ACL ruptures than simple differences in anterior tibial translation. However, in clinical practice, the physical examination and the KT-1000 arthrometric evaluation are the most commonly used methods for physicians for the diagnosis of ACL ruptures. Although the scar pattern of the ACL remnant to the notch or posterior cruciate ligament and chronicity may contribute to knee stability ${ }^{5,8)}$, we did not differentiate between chronic and acute injuries or different scar patterns. According to Nakamae et al. ${ }^{8}$, chronicity up to one year after injury may contribute to anteroposterior knee stability but not to rotational stability. Although not related directly with the aim of this study, the final decision to preserve tissue and perform an augmentation technique was also subjective and was performed according to the surgeon's experience and criteria.

It is not easy to decide whether to preserve a bundle. The suggested predictive score can be used to summarize the three most clinically relevant tests to diagnose complete or partial ACL ruptures. According to our findings, it is highly recommended to perform EUA and arthrometric evaluation prior to ACL reconstruction. We think that the suggested prognostic score can be a significant additional tool for the surgeon to decide if an ACL bundle is functional, and whether to preserve it and perform augmentation instead of complete ACL reconstruction.

\section{Conclusions}

The Lachman test, pivot shift test and arthrometric assessment under anesthesia can be useful for diagnosis of partial ACL tears. A composite score of less than five from the tests suggests a partial ACL rupture.

\section{Conflict of Interest}

No potential conflict of interest relevant to this article was reported.

\section{References}

1. Zantop T, Herbort M, Raschke MJ, Fu FH, Petersen W. The role of the anteromedial and posterolateral bundles of the anterior cruciate ligament in anterior tibial translation and internal rotation. Am J Sports Med. 2007;35:223-7.

2. Ochi M, Adachi N, Deie M, Kanaya A. Anterior cruciate ligament augmentation procedure with a 1-incision technique: anteromedial bundle or posterolateral bundle reconstruction. Arthroscopy. 2006;22:463.

3. Serrano-Fernandez JM, Espejo-Baena A, Martin-Castilla B, De La Torre-Solis F, Mariscal-Lara J, Merino-Ruiz ML. Augmentation technique for partial ACL ruptures using semitendinosus tendon in the over-the-top position. Knee Surg Sports Traumatol Arthrosc. 2010;18:1214-8.

4. Sonnery-Cottet B, Lavoie F, Ogassawara R, Scussiato RG, Kidder JF, Chambat P. Selective anteromedial bundle recon- 
struction in partial ACL tears: a series of 36 patients with mean 24 months follow-up. Knee Surg Sports Traumatol Arthrosc. 2010;18:47-51.

5. Crain EH, Fithian DC, Paxton EW, Luetzow WF. Variation in anterior cruciate ligament scar pattern: does the scar pattern affect anterior laxity in anterior cruciate ligamentdeficient knees? Arthroscopy. 2005;21:19-24.

6. Adachi N, Ochi M, Uchio Y, Sumen Y. Anterior cruciate ligament augmentation under arthroscopy: a minimum 2-year follow-up in 40 patients. Arch Orthop Trauma Surg. 2000;120:128-33.

7. Adachi N, Ochi M, Uchio Y, Iwasa J, Ryoke K, Kuriwaka M. Mechanoreceptors in the anterior cruciate ligament contribute to the joint position sense. Acta Orthop Scand. 2002;73: 330-4.

8. Nakamae A, Ochi M, Deie M, Adachi N, Kanaya A, Nishimori M, Nakasa T. Biomechanical function of anterior cruciate ligament remnants: how long do they contribute to knee stability after injury in patients with complete tears? Arthroscopy. 2010;26:1577-85.

9. Lane CG, Warren R, Pearle AD. The pivot shift. J Am Acad Orthop Surg. 2008;16:679-88.

10. Bach BR Jr, Warren RF, Flynn WM, Kroll M, Wickiewiecz TL. Arthrometric evaluation of knees that have a torn anterior cruciate ligament. J Bone Joint Surg Am. 1990;72:1299306.

11. Ochi M, Adachi N, Uchio Y, Deie M, Kumahashi N, Ishika- wa M, Sera S. A minimum 2-year follow-up after selective anteromedial or posterolateral bundle anterior cruciate ligament reconstruction. Arthroscopy. 2009;25:117-22.

12. Fritschy D, Panoussopoulos A, Wallensten R, Peter R. Can we predict the outcome of a partial rupture of the anterior cruciate ligament? A prospective study of 43 cases. Knee Surg Sports Traumatol Arthrosc. 1997;5:2-5.

13. Bak K, Scavenius M, Hansen S, Norring K, Jensen KH, Jorgensen $\mathrm{U}$. Isolated partial rupture of the anterior cruciate ligament. Long-term follow-up of 56 cases. Knee Surg Sports Traumatol Arthrosc. 1997;5:66-71.

14. Sonnery-Cottet B, Barth J, Graveleau N, Fournier Y, Hager JP, Chambat P. Arthroscopic identification of isolated tear of the posterolateral bundle of the anterior cruciate ligament. Arthroscopy. 2009;25:728-32.

15. Dejour D, Ntagiopoulos PG, Saggin PR, Panisset JC. The diagnostic value of clinical tests, magnetic resonance imaging, and instrumented laxity in the differentiation of complete versus partial anterior cruciate ligament tears. Arthroscopy. 2013;29:491-9.

16. Yoon KH, Bae DK, Cho SM, Park SY, Lee JH. Standard anterior cruciate ligament reconstruction versus isolated singlebundle augmentation with hamstring autograft. Arthroscopy. 2009;25:1265-74.

17. Liu W, Maitland ME, Bell GD. A modeling study of partial ACL injury: simulated KT-2000 arthrometer tests. J Biomech Eng. 2002;124:294-301. 